\title{
Are we addressing the nutritional needs of hospital inpatients who are nil by mouth?
}

\author{
L. Menzies, L. Neave and S. Mann \\ Barnet and Chase Farm NHS Trust, Barnet Hospital, Wellhouse Lane, Barnet EN5 3DJ, UK
}

The cost of malnutrition and associated disease has been estimated to be more than $£ 7.3$ billion in 2003 ; the majority of this expenditure was due to treatment of malnourished patients in hospital and long-term care $(£ 3.8 \text { billion })^{(1)}$. Given this profound economic impact and the emergence of evidence that improving patient nutrition can reduce hospital stay and complications, national campaigns have raised awareness of malnutrition in an attempt to reduce its prevalence in UK hospitals ${ }^{(2,3)}$.

National guidance includes risk assessment for malnutrition for all new hospital admissions. Patients at risk of malnutrition include those who have eaten little or nothing for five or more days or are likely to eat little or nothing in the following $5 \mathrm{~d}^{(4)}$. We considered that inpatients who are 'Nil by Mouth' (NBM) represent a particularly high risk cohort for malnutrition. The aims of our study included assessment of (i) the prevalence and duration of NBM, (ii) whether the indication was appropriate, (iii) whether these patients had been screened for malnutrition risk on admission, (iv) whether alternative nutritional supplementation had been arranged and (v) whether the Nutrition Multidisciplinary Team were adequately involved in patient care. We audited four wards (two general medical, one acute stroke unit and one surgical) within Barnet Hospital on two separate occasions, encompassing 192 patients in total.

\begin{tabular}{|c|c|c|c|}
\hline \multicolumn{2}{|l|}{ Sample Characteristics } & \multicolumn{2}{|c|}{ Adequate documentation in NBM patients } \\
\hline Percentage of patients NBM & 9.9 & Weight & $73 \%$ \\
\hline Mean age of NBM patients & 75 years (SD 17.7 years) & BMI & $68 \%$ \\
\hline NBM mean duration & $8.7 \mathrm{~d}(\mathrm{SD} 8.6 \mathrm{~d})$ & Weight loss over past $3-6$ months & $32 \%$ \\
\hline NBM duration $\geq 5 \mathrm{~d}$ & $53 \%$ & Nutrition risk score sheet started & $78 \%$ \\
\hline \multicolumn{2}{|c|}{ Indication for NBM: appropriate in all cases, most commonly unsafe swallow, e.g., after stroke } & $\begin{array}{l}\text { Nutrition risk score sheet completed } \\
\text { in } \leq 1 \mathrm{~d} \text {, with weight and BMI recorded }\end{array}$ & $35 \%$ \\
\hline
\end{tabular}

We recorded the time from when a patient was made NBM until the advent of alternative nutritional replacement, with $42 \%$ of patients failing to receive optimal alternative supplementation as per best practice guidelines. We also identified delays in the involvement of speech and language therapists and dietitians in the care of some patients who were NBM, finding this to be associated with a delay in the provision of alternative nutritional replacement in $32 \%$ of patients.

Following our analysis, a number of clinical practice recommendations were considered; for example methods for improvement of (i) risk screening, (ii) more prompt involvement of the Nutrition Multidisciplinary Team and (iii) documentation of nutritional parameters in the medical notes. Together with our key findings, these were disseminated in the following ways; (i) presentation and discussion at the quarterly Nutrition Steering Committee Meeting, (ii) presentation at the Medical Grand Round and (iii) design of a promotional leaflet for nursing staff, distributed by hospital matrons.

In conclusion, in a district general hospital, a significant proportion of inpatients are made NBM, particularly the elderly. Often they are NBM for more than $5 \mathrm{~d}$, with variable delays in both obtaining assessment from dietitians and speech and language therapists and providing alternative nutritional replacement to meet their needs. We have therefore recommended that an IR 1 form is completed if a patient is NBM for five or more days without provisional of alternative means of nutritional support.

1. Elia M, Stratton RJ, Russell C et al. (2006) The cost of disease-related malnutrition in the UK and economic considerations for the use of oral nutritional supplements in adults. BAPEN.

2. Department of Health (2007) Improving Nutritional Care: A Joint Action Plan from the Department of Health and Nutrition Summit Stakeholders. Department of Health Publications.

3. Council of Europe Resolution Food and Nutritional Care in Hospitals (2007) Ten key characteristics of good nutritional care in hospitals.

4. National Collaborating Centre for Acute Care (2006) Nutrition support in adults Oral nutrition support, enteral tube feeding and parenteral nutrition. Commissioned by the National Institute of Clinical Excellence. 